\title{
AUTOMATIC WHITE BALANCE FOR DIGITAL STILL CAMERA
}

\author{
Yung-Cheng Liu, Wen-Hsin Chan and Ye-Quang Chen \\ Industrial Technology Research Institute \\ ITRI/OES, 195-8, Sec. 4, Chung-Hsing Rd. \\ Chutung 310, Taiwan, ROC
}

\begin{abstract}
A new algorithm of automatic white balance for electronic images has been developed. This algorithm uses fuzzy logic rules for determination of the color parameters in order to minimize the color temperature difference of various light sources. This algorithm has been implemented in a newly designed electronic still camera, and resulted in highly improved image quality.
\end{abstract}

\section{Introduction}

An object may appear different in color when it is illuminated with different light sources. This is due to the color temperature difference of the light sources, which induces the shift of the reflection spectrum of the object from the "true" color. When a white object is illuminated with low color temperature light source, the reflection becomes reddish. On the other hand, the high temperature light source causes bluish in color to the same white object. The human vision may not be able to distinguish the color difference due to the "color constancy"l of human eyes. However, for an image recording media such as photographic films or video tapes, the reflected light of an object from a given light source will be recorded, and results in a color difference, if the light source is not standard.

To compensate this color difference caused by various light sources, a white balance mechanism is employed in most electronic cameras. Usually, a color video signal from a sensor can be decomposed into red, green, and blue components, or other components in color space. The signal of each color can have its own amplifier. To adjust these amplifiers with different gains, the composite color can be corrected to near " true" color.

For white balance adjustment, the traditional mechanism in an electronic camera uses color sensors to measure the color intensity of the environmental illumination. The functional block diagrams for two and three sensor systems are shown in the Fig. 1 and Fig. 2 respectively. The two-sensor system uses sensors with red and blue optical filters for detection and measurement. The output signal of each sensor passes a logarithm pre-amplifier. The ratio of these two color signals is obtained by a divider (CMP), then it is digitized after an A/D converter. Using a table stored in a ROM (read only memory), this digitized ratio is converted to corresponding gain factors for the image color adjustment.

The three-sensor system uses red, green, and blue sensors for color measurement. The ratio of red to green is employed for red color gain control, and the ratio of blue to green is for blue color gain control. As a result, a normal color of digital image can be obtained by this mechanism. 
In addition to the color sensor systems, another method is to use the image sensor (usually $\mathrm{CCD}$, charge-coupled devices) as a color sensor for color measurement. In an analog electronic camera, the output signal of a CCD can be decomposed into the luminance $Y$, and the chrominance $\mathrm{R}-\mathrm{Y}$ and B-Y. The integrated values of both $R-Y$ and $B-Y$ over one image frame can be calculated. These values are used for the controls of red and blue color amplifiers. The functional block diagram of this system is shown in Fig. 3.

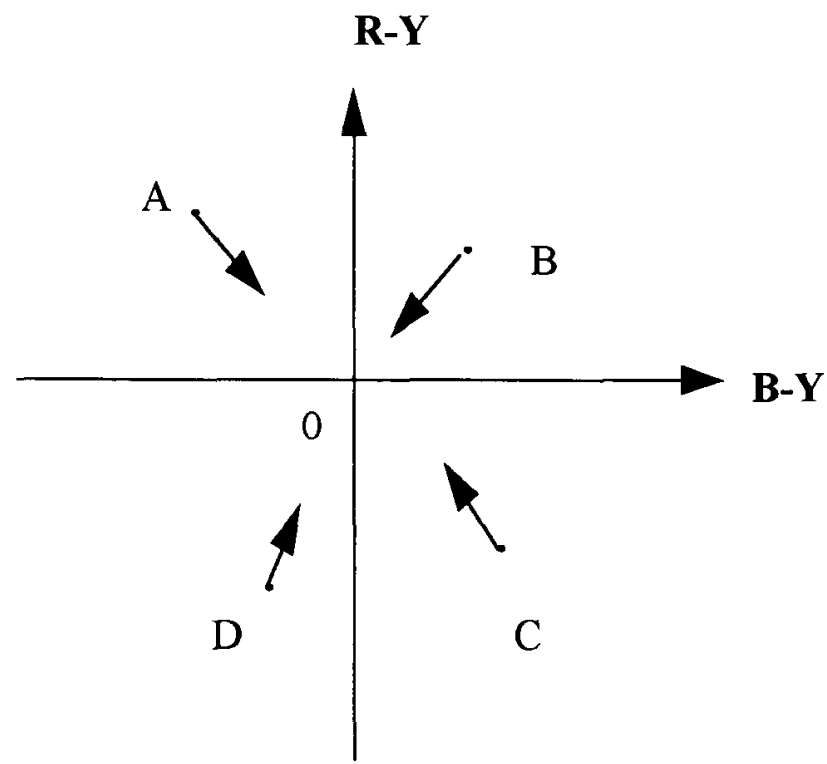

Fig. 4 the (R-Y)-(B-Y) 2-dimension coordinate

The integrated values of both $\mathrm{R}-\mathrm{Y}$ and $\mathrm{B}$ $\mathrm{Y}$ can be plotted on a R-Y, B-Y coordinate system. Fig. 4 shows an example of four different types of $\mathrm{A}, \mathrm{B}, \mathrm{C}$, and $\mathrm{D}$ points. The deviations of these points from the origin $\mathrm{O}$, which is at the white balance, need to be corrected in the arrow direction in order to approximate the $\mathrm{O}$ point. This method is under the assumption that the integrated values of a color picture are at white color. The bias toward blue or red color is introduced by the color temperature of a light source. However, when a large object or background with a uniform color, the color compensation may cause the loss of integrity of the color.
The following sections will describe an automatic white balance algorithm used in a digital still camera. This method will reduce the effect of a large object with uniform color in an image field of view. The fuzzy theory is employed for the color gain determination, therefore experiments are implemented to get statistic results. These experiments are described in Section 2. The results and concluded fuzzy rules are discussed, and the algorithm based on those fuzzy rules in a digital electronic camera is described. Section 3 gives the testing results of the algorithm. The conclusion of this study is given in Section 4.

\section{Experiment}

A CCD based camera was used for this experiment. The digital signals of a color image can be decomposed into three components: Y, $\mathrm{Cr}$, and $\mathrm{Cb}$; where $\mathrm{Y}$ is the intensity, and $\mathrm{Cr}, \mathrm{Cb}$ are the color components, according to CCIR601 standard. The pixel data $\mathrm{Y}, \mathrm{Cr}, \mathrm{Cb}$ are accumulated over an image frame. Objects with various colors were illuminated with difference light sources. The result data are plotted on $\mathrm{Cr}$ Cb coordinates.

The light source used in this experiment and their codes are listed in the table 1.

\begin{tabular}{|l|c|c|c|c|c|}
\hline CODE & H & D & I & C & UV \\
\hline $\begin{array}{l}\text { Light } \\
\text { Source }\end{array}$ & Horizon & Daylight & $\mathrm{INC}_{\mathrm{A}}$ & $\begin{array}{l}\text { Cool } \\
\text { White }\end{array}$ & $\begin{array}{l}\text { Ultraviolet } \\
\text { Rays }\end{array}$ \\
\hline
\end{tabular}

Table 1. light source names and their codes

Any uniform color can be represented with a point on the $\mathrm{Cb}-\mathrm{Cr}$ coordinate system. A monochromatic object illuminated with a standard light source will be on a nominal position on the $\mathrm{Cb}-\mathrm{Cr}$ coordinate. However, with different light sources or light intensity, the position will deviate toward $\mathrm{Cb}$ (indicate bluish) 


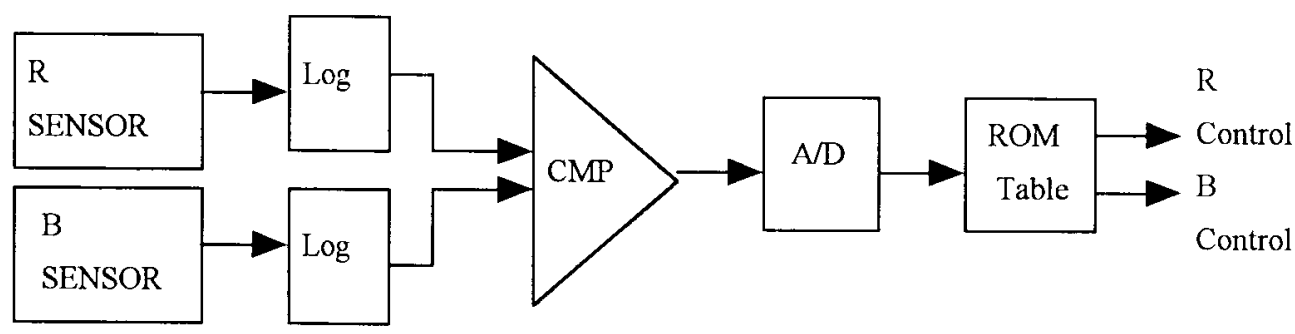

Fig. 1 two-color sensor detector

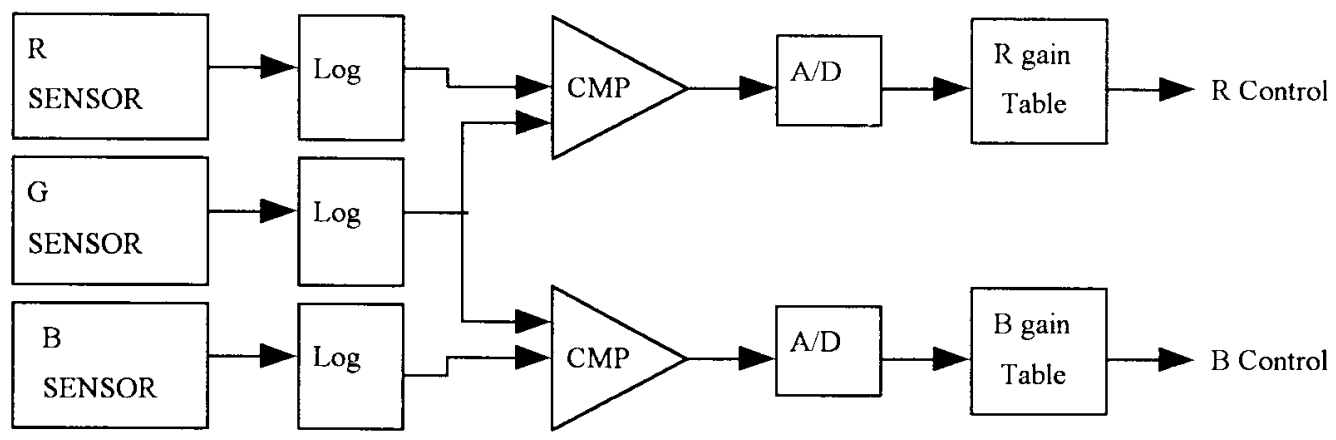

Fig. 2 three-color sensor detector

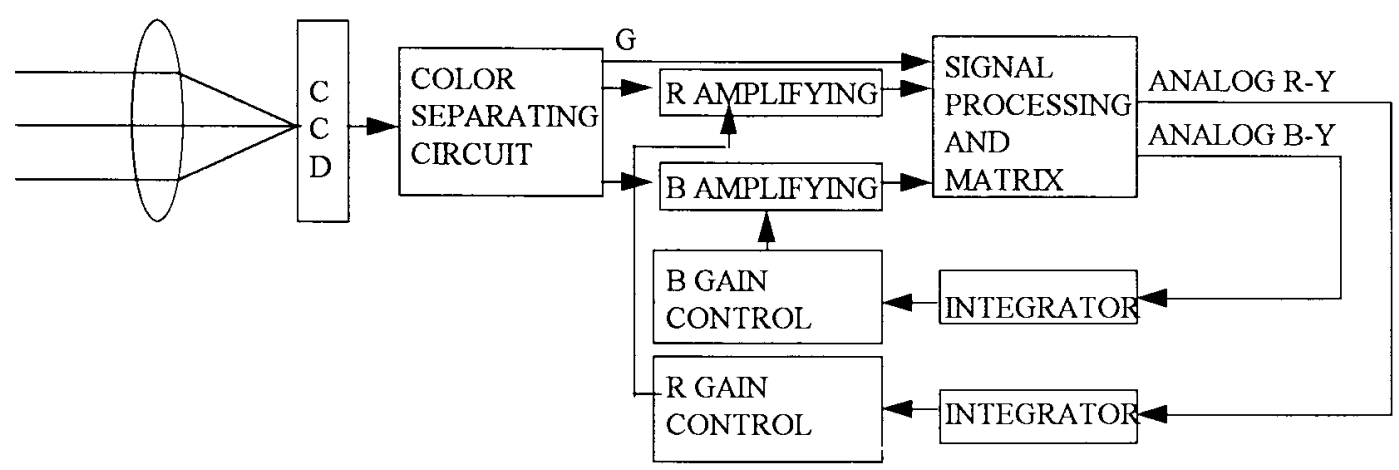

Fig. 3 automatic white balance in analog electronic camera

The above white balance circuits have been used for electronic cameras for years, but there are the following drawbacks:

1) The spectral responsibility of a color sensor is different from image sensor, hence the color compensation is limited.

2) Usually a color sensor has limited dynamic range, therefore it is not effective in bright or dim environment.
3) The analog signal processing prior to the $A / D$ converter may be effected by the temperature variation. The calibration may be required.

4) An extra room is required to house the sensors in camera assembly.

5) The view angle of color sensor should be close to the camera lens. This will introduce extra design effort. 


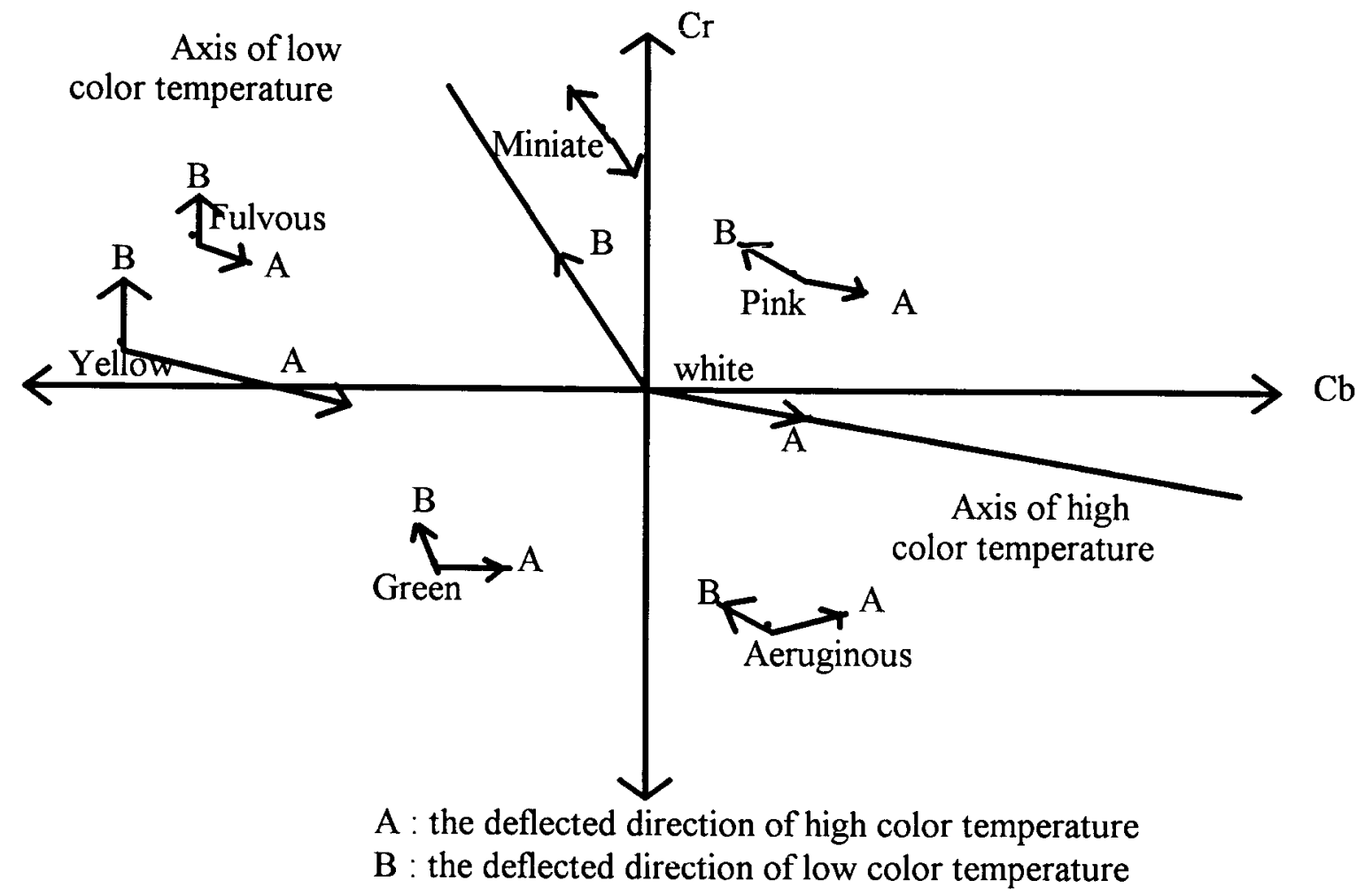

Fig. 5 the experimental data of colors under different light sources.

or $\mathrm{Cr}$ (reddish) coordinate. Some of the experimental results are summarized in the Figure 5.

The experimental results depict that:

1) A dark color has less deviation from nominal position under different light sources, as opposed to the bright color, where the deviation is significant on the $\mathrm{Cb}$ and $\mathrm{Cr}$ components;

2) When a white object is illuminated with different light sources, the slope of deflection, i.e. the ratio of $\mathrm{Cr}$ to $\mathrm{Cb}, \mathrm{r}=\mathrm{Cr} / \mathrm{Cb}$, is about between -1.5 and -0.5 ;

3) At high luminance, the color components are easy to be saturated; while at low luminance, the color components become colorless.

The automatic white balance algorithm has been developed. A frame of image captured with a CCD camera can be divided into 8 segments for weightings. Fig.6 shows two types of segmentation. The averages of $\mathrm{Cr}$ and $\mathrm{Cb}$ components of all pixels within each segment are calculated, and then weighted under fuzzy control means. Based on experimental results, the fuzzy rules were described below:

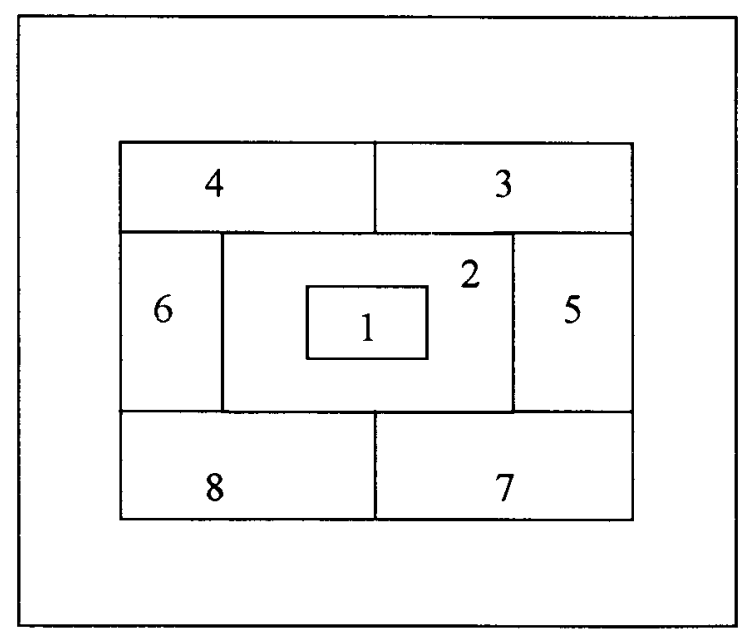

Fig.6a division of frame (I) 


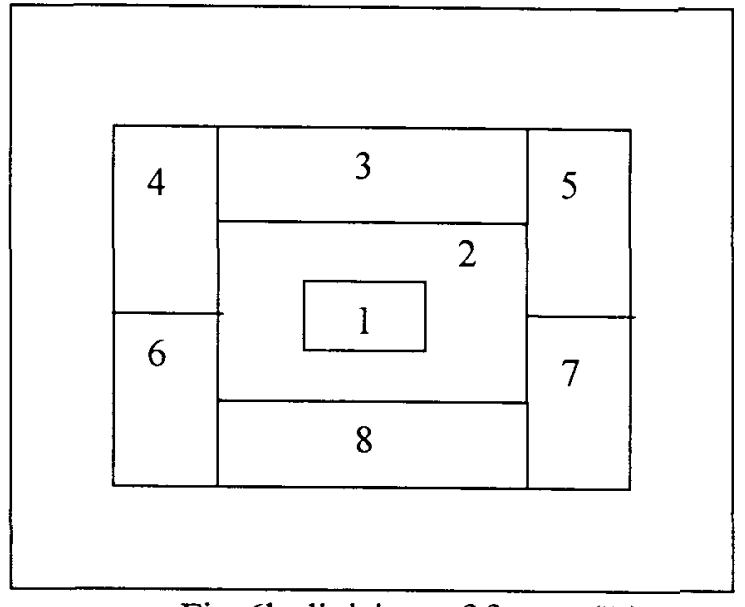

Fig.6b division of frame (II)

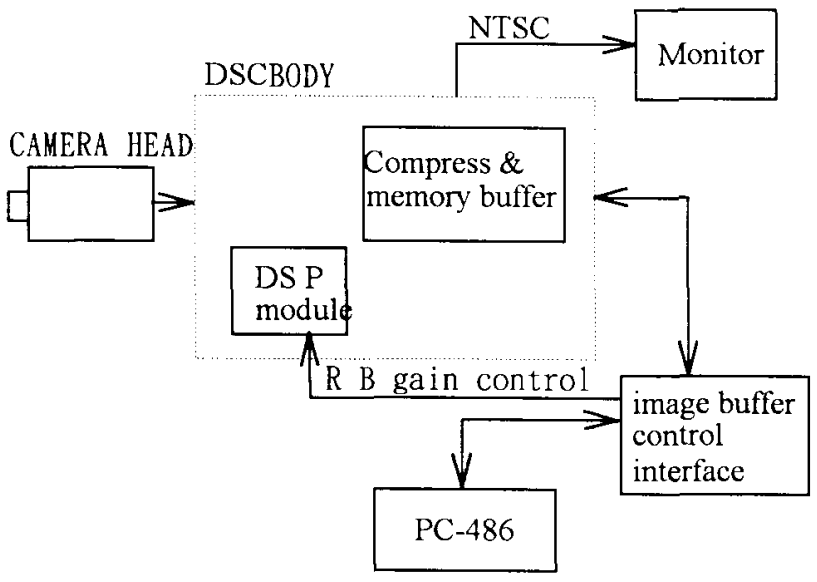

Fig.7 a simulation block diagram of automatic white balance

1) At high luminance, the color components are easy to be saturated, while at low luminance, the color components become colorless. Hence, the $\mathrm{Cb}$ and $\mathrm{Cr}$ will be weighted with small value under the conditions of high end and low end luminance.

2) The averages of $\mathrm{Cb}$ and $\mathrm{Cr}$ for each segment are weighted less for dark colors than bright colors.

3 ) When a large object or a background occupies more than one segment, its color will dominate that segment. The $r$ value will be similar among adjacent segments. In this case, the given weighting for that segment is small in order to avoid over compensation on the color of the picture. Otherwise, if $r$ is very different from the adjacent segments, and is about between -1.5 to -0.5 , the given weighting is large.

Fig. 7 is the block diagram of a simulation for the development and testing of an automatic white balance algorithm. This simulation system consists of a camera head, a digital still camera (DSC), a monitor, an image buffer, and a 486 personal computer. The camera head and the DSC were designed in our laboratory. The image is captured by a CCD area array. The output signals from $\mathrm{CCD}$ are converted into 10 bits' digital data. Those raw data are then processed with a DSP/IC (digital signal processor integrated circuits) to implement color conversion, interpolation, and coding. The result digital output consists of $\mathrm{Y}, \mathrm{Cb}, \mathrm{Cr}$ components for each pixel. The full image frame is then stored in an image buffer. The testing algorithm is executed and evaluated in the $486 \mathrm{PC}$

As discussed before, the image is divided into 8 segments. The averages of $\mathrm{Cb}$ and $\mathrm{Cr}$ for each segment are calculated. Then the weighting factors for each segment are determined, based on the fuzzy control means, to calculate the evaluating $\mathrm{Cb}^{\prime}$ and $\mathrm{Cr}^{\prime}$ of the whole image frame The $\mathrm{Cb}^{\prime}$ and $\mathrm{Cr}^{\prime}$ indicate the deviation of the image color from the white balance point. These values are employed to obtain the gains for $\mathrm{Cb}$ and $\mathrm{Cr}$ of each pixel. The averages of adjusted $\mathrm{Cb}$ and $\mathrm{Cr}$ are calculated. If the result is not close to the white balance point, the iteration will be performed. Fig. 8 is a flow chart of the white balance algorithm using fuzzy control means.

\section{Result}

During the experiment and the development of the algorithm, the $\mathrm{Cb}$ and $\mathrm{Cr}$ 
values showed a strong correlation with the color temperature. As expected, the low color temperature gives reddish image, while the high color temperature gives bluish image.

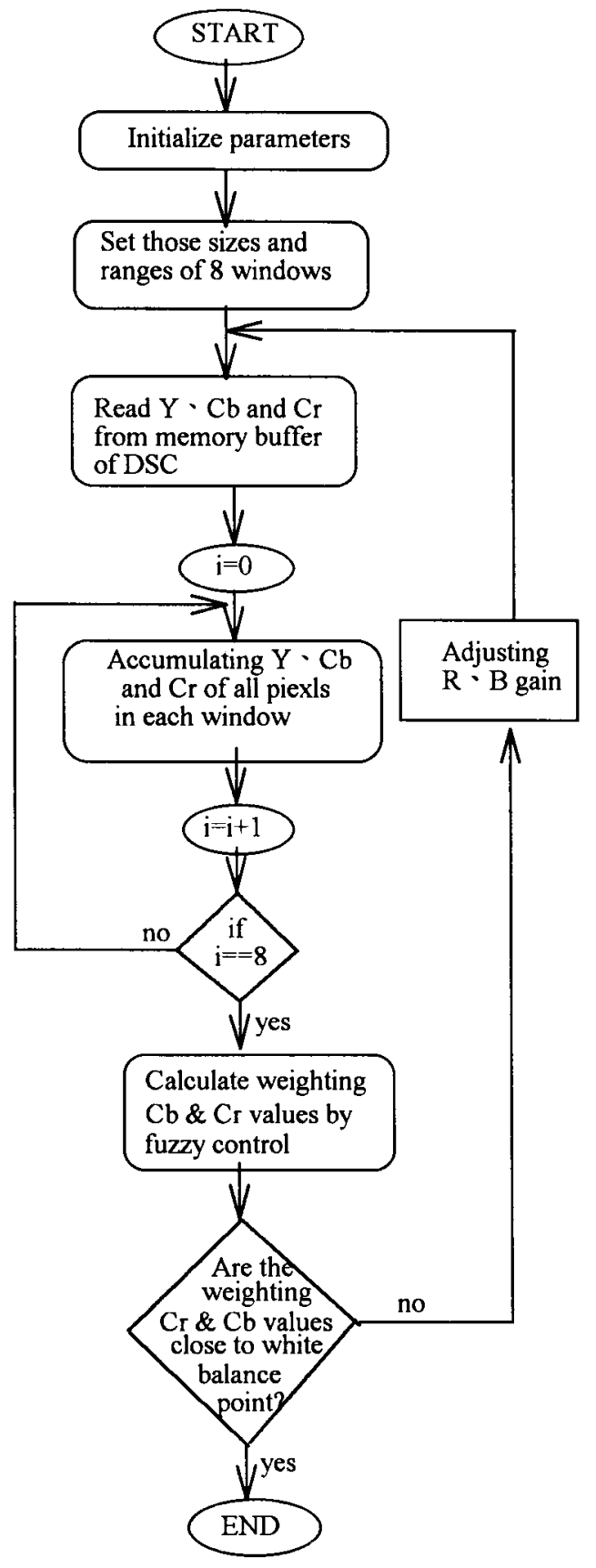

Fig. 8 flow chart of white balance by fuzzy control means.

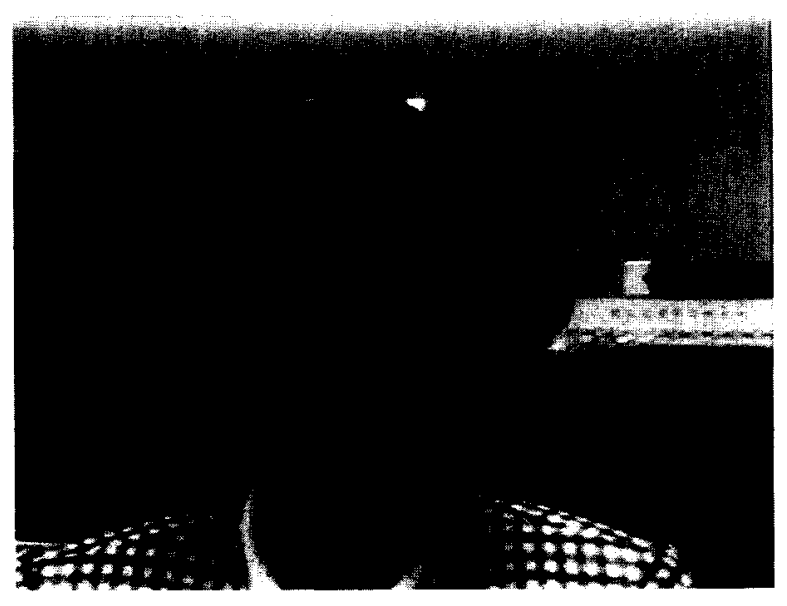

Fig.9a an uncorrected image

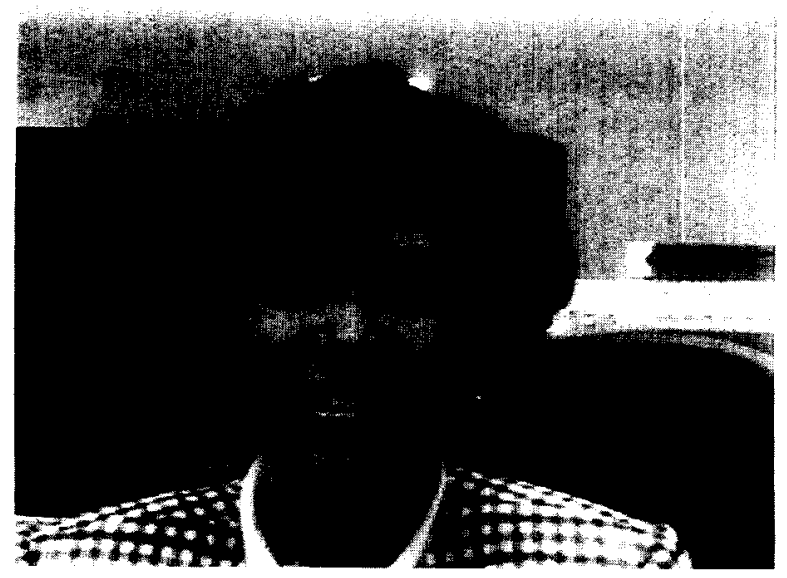

Fig. $9 \mathrm{~b}$ a corrected image

Fig. $9 \mathrm{a}$ is a picture exposed under low color temperature. The color is reddish. Using the automatic white balance algorithm, the color is corrected as shown in Fig. $9 \mathrm{~b}$. The picture was divided into 8 segments, The 8 sets of $\mathrm{Cb}, \mathrm{Cr}$ averages are plotted on a $\mathrm{Cb}-\mathrm{Cr}$ coordinate system (Fig 10a, symbol "."). After weighting for each segment, based on the fuzzy control mean, the $\mathrm{Cb}^{\prime}$ and $\mathrm{Cr}^{\prime}$ were obtained and is plotted with a symbol of " $\mathrm{x}$ ". The deviation is used for $\mathrm{Cb}$ and $\mathrm{Cr}$ gain adjustments for all pixels. The averages of adjusted $\mathrm{Cb}, \mathrm{Cr}$ for each segment were calculated again, and the induced $\mathrm{Cb}^{\prime}$ and $\mathrm{Cr}^{\prime}$ were obtained. The result (Fig 10b) shows that the $\mathrm{Cb}$ and $\mathrm{Cr}$ for each segment shift toward the origin, and the final $\mathrm{Cb}^{\prime}$ and $\mathrm{Cr}^{\prime}$ are 
very close to white balance point. The improvement on the picture in color is significant (Fig. 9b).

\section{Conclusion}

This automatic while balance feature has been included in a newly designed digital camera prototype. The algorithm was implemented with FPGA circuit in order to have real time processing. The performance on white balance is significantly improved. This method uses the picture segments with different weightings, which minimize the color effect of large objects and background. The fuzzy control rules were obtained with experimental data. Therefore, using these rules for gain determination gives the optimal performance on white balance.

\section{Biography}

Yung-Cheng Liu was born in Chang-Hwa, Taiwan R.O.C., in 1965. He received his B.S. degree in electronics at Chung-Yuan Christian University, in 1988, and then received his M.S. degree in Institute of Optical Science at National Central University, in 1990. He is a member of the Chinese Image Processing and Pattern Recognition Society, and is with OptoElectronics \& System Lab. of Industrial Technology Research Institute since 1990. His research interests include the development of Opto-electronic system, image processing and digital video camera system.

Wen-Hsin Chan received his $\mathrm{Ph}$. D. degree in Electrical and Computer Engineering from State University of New York at Buffalo in 1984. From 1984 to 1985, he was a senior engineer with the Remote Sensing Group at Lockheed Aerospace Company. From 1985 to 1992, He was a staff engineer with Guidance System Division at Allied-Signal Bendix Inc. Since 1992, he has been at the Industrial Technology Research Institute in Taiwan, where he has served as a deputy director in the

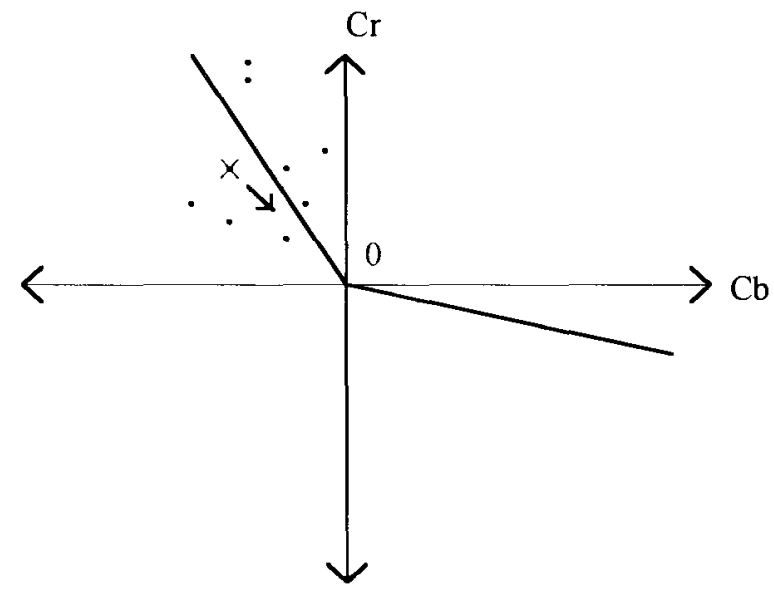

Fig. 10a

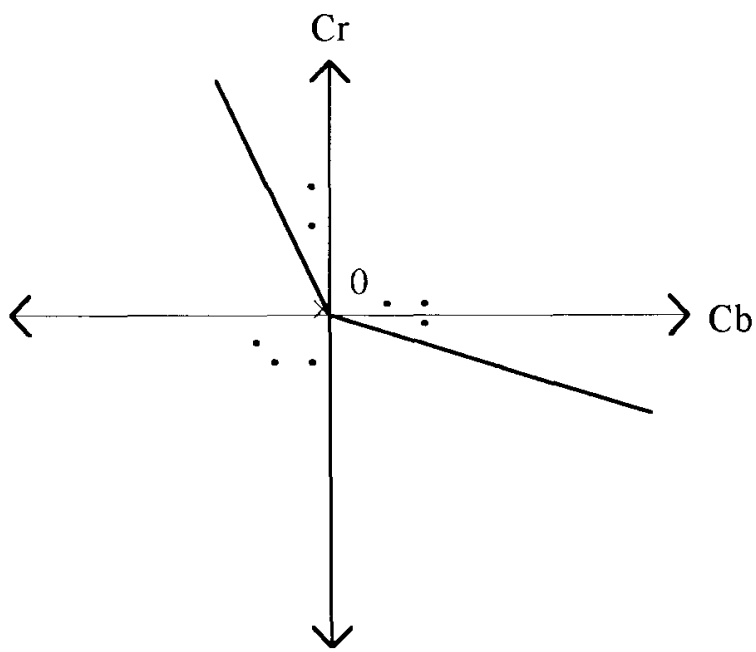

Fig. 10b

Planning and Promotion Division, and currently as a manager in the image processing department.

\section{Reference}

1) Peter Gouras, "The Perception of Colour", New York, USA, 1991.

2) A. Kaufmann, "Introduction to the Theory of Fuzzy Subsets", Orlando, Florida, 1975.

3 ) George J. Klir and Tina A. Folger, "Fuzzy Sets, Uncertainty and Information", Englewood Cliffs, New Jersey, 1988. 